\title{
The effect of clouds on the dynamical and chemical evolution of gas-rich dwarf galaxies
}

\author{
S. Recchi $^{1, \star}$ and G. Hensler ${ }^{1, \star \star}$ \\ Institute of Astronomy, University of Vienna, Türkenschanzstrasse 17, A-1180 Vienna, Austria \\ The dates of receipt and acceptance should be inserted later
}

\begin{abstract}
Key words hydrodynamics - galaxies: abundances - galaxies: dwarf - galaxies: evolution - ISM: clouds - ISM: jets and outflows

We study the effects of clouds on the dynamical and chemical evolution of gas-rich dwarf galaxies, in particular focusing on two model galaxies similar to IZw18 and NGC1569. We consider both scenarios, clouds put at the beginning of the simulation and continuously created infalling ones. Due to dynamical processes and thermal evaporation, the clouds survive only a few tens of Myr, but during this time they act as an additional cooling agent and the internal energy of cloudy models is typically reduced by $20-40 \%$ in comparison with models without clouds. The clouds delay the development of large-scale outflows, therefore helping to retain a larger amount of gas inside the galaxy. However, especially in models with continuous creation of infalling clouds, their bullet effect can pierce the expanding supershell and create holes through which the superbubble can vent freshly produced metals. Moreover, assuming a pristine chemical composition for the clouds, their interaction with the superbubble dilutes the gas, reducing the metallicity (by up to $\sim 0.4$ dex) with respect to the one attained by diffuse models.
\end{abstract}

(C) 2009 WILEY-VCH Verlag GmbH \& Co. KGaA, Weinheim

\section{Introduction}

Intense episodes of star formation (SF) in dwarf galaxies (DGs) are associated to the development of large-scale outflows. These have enormous multiple effects on the evolution of DGs: the hot gas carries off a large amount of freshly produced metals, thermalizes the interstellar medium (ISM), reduces the gas content, ceases the SF, and further more. The study of this phenomenon through numerical simulations has therefore been in the focus of several authors in the past. The overall picture is that the occurrence of outflows is initially driven by the thermal pressure of a hot, high pressurized gas and is favored by a flat distribution of the ISM, which allows an easy vertical transport of material, whereas horizontal transport is very limited. Therefore, outflows are able to eject a large fraction of freshly produced metals but only a very limited fraction of ISM (D'Ercole \& Brighenti 1999; Recchi, Matteucci \& D’Ercole 2001).

Most of these studies, however, consider a single-phase ISM, although attempts to perform multiphase hydrodynamical simulations have been made in the past, particularly using the so-called chemodynamical approach (Samland, Hensler \& Theis 1997; Hensler 2003; Hensler, Theis \& Gallagher 2004). In two previous papers, we have studied the dynamical and chemical evolution of model galaxies similar to IZw18 (Recchi et al. 2004, hereafter Paper I) and NGC1569 (Recchi et al. 2006). In this work we simulate the same two galaxies, but we increase arbitrarily the gas density of some specific regions of the computational grid, in order to create

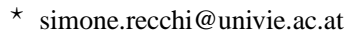

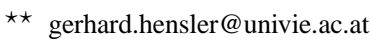

a "cloudy" phase, and we address the question how and to which extent this new component alters the former results.

It is particularly interesting to compare the chemical evolution of models with and without clouds. In fact, clouds are expected to dilute the hot metal-rich gas through evaporation, allowing for a reduction of the metallicity without altering the abundance ratios (Köppen \& Hensler 2005). On the other hand, the clouds can act as a cap and hamper the development of a galactic wind by means of their drag and, if they are dispersed, due to Kelvin-Helmholtz instability and evaporation of mass load to the hot outflow. As a result these should lead to an increase of the metallicity of the galactic ISM, since, as we have seen, galactic winds otherwise carry metal-enriched gas away from the galaxy. In this case, the abundance ratios are affected if the ejection efficiencies depend on the different chemical species.

\section{The model}

The simulations are performed by means of a 2-D chemodynamical code described in Paper I and references therein. It assumes cylindrical symmetry and follows in detail the release of mass, individual elements and energy from SNeIa, SNeII and stellar winds. As mentioned in the introduction, the initial set-up of the models is aimed at reproducing the main characteristics of two well-studied gas-rich dwarf galaxies, namely IZw18 and NGC1569.

The cloudy phase is introduced by randomly choosing grid cells and increasing artificially the density therein and in the neighboring cells in order to reproduce a cloud density profile similar to the observed ones (namely $\rho_{\mathrm{cl}} \propto R_{\mathrm{cl}}^{-1.7}$, 


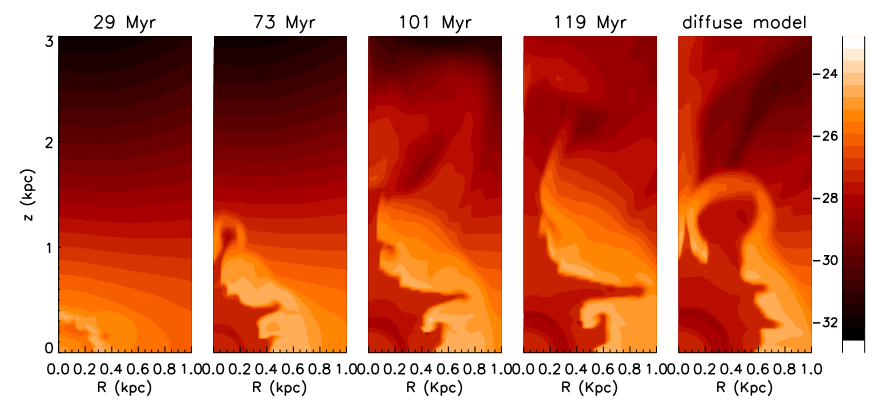

Fig. 1 Density contours of the gas for the static cloudy model at 4 evolutionary times (labelled, in Myr, on the top of each panel). The logarithmic density scale (in $\mathrm{g} \mathrm{cm}^{-3}$ ) is on the right-hand strip. For reference, also the density contours of the correspondent diffuse model at $t \sim 120 \mathrm{Myr}$ is displayed (rightmost panel).

where $R_{\mathrm{cl}}$ is the distance from the center of the cloud; de Heij, Braun \& Burton 2002). We assume that the chemical composition of the clouds is pristine (i.e. without metals). The clouds can be either added at the beginning of the simulation (static cloudy models) or continuously created during the evolution of the model. In the latter case, we will give also an infall velocity of $10 \mathrm{~km} \mathrm{~s}^{-1}$ to the clouds (infall cloudy models). We will also consider as comparison models obtained without a cloudy phase (diffuse models).

Since saturated heat conduction is included (Cowie \& McKee 1977) in the static cloudy model the clouds can only act until they vanish due to evaporation, while in the infall cloudy model clouds are reborn on timescales of a few Myr.

\section{Results}

\subsection{Dynamical evolution}

The snapshots of the evolution of a prototypical static cloudy model are shown in Fig. 11(first 4 panels) together with the density distribution on the analogous diffuse model at $t \sim$ $120 \mathrm{Myr}$ (rightmost panel). This model is aimed at reproducing IZw18 and has a SF history characterized by a mild, long-lasting episode of SF and a recent burst (see Paper I; Recchi \& Hensler 2007 for details). The clouds are soon engulfed in the hot cavity and evaporate on a short timescale of a few tens of Myrs. The presence of the clouds is however sufficient to create large shears and eddies. Due to these structures and due to the evaporation of the clouds within the superbubble, radiative losses in this model are significantly larger than in the equivalent diffuse model (by $\sim 20$ $\%)$.
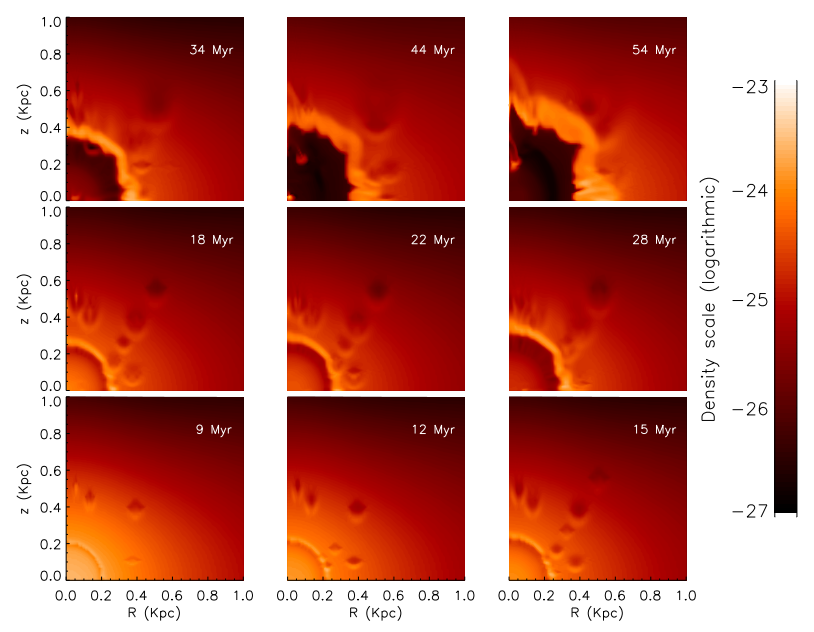

Fig. 2 Density contours for the infall cloudy model at 9 evolutionary times (labeled, in Myr, at the top right corner of each panel). The logarithmic density scale (in $\mathrm{g} \mathrm{cm}^{-3}$ ) is on the right-hand strip.

At the beginning therefore the evolution of the superbubble is slightly slower than in diffuse models. In particular, a galactic outflow starts $\sim 75 \mathrm{Myr}$ after the beginning of the SF; 5-10 Myr later than the analogous diffuse model. However, the presence of clouds strongly distorts the shape of the supershell and the highly pressurized gas inside the cavity can more easily find regions of lower pressure, from which it is easy to pierce the shell and break out. This creates the tongues visible in Fig. 1. From these tongues freshly produced metals can leave the galaxy. Therefore, in spite of the (slightly) slower development of the galactic wind, the static cloudy model maintains a large ejection efficiency of newly synthesized metals.

A similar behavior is seen in the infall cloudy model (Fig. 2). This model has the same setup as the static cloudy model seen above, the only difference being the treatment of clouds. Bow shocks created by the clouds while falling towards the disk are evident in this figure. The interaction between clouds and ISM produces also Kelvin-Helmholtz instabilities and evaporation, therefore, also in this model, clouds do not survive more than a few tens of Myr. The radiative losses are more significant than in the static cloudy model because continuously formed clouds deliver more mass to the outflow. At the end of the simulation the thermal budget of the system is reduced by $\sim 35 \%$ compared to the analogous diffuse model. Consequently, the first weak signs of a galactic outflow appear only $\sim 90 \mathrm{Myr}$ after the beginning of the SF; $\sim 20-25$ Myr later than in the diffuse model. However, analogously to the case of the static cloudy model, the ram pressure of the clouds pierces the supershell and creates holes through which hot cavity gas (and freshly produced metals) can leak out. 


\subsection{Chemical evolution}

The evolution of the mean oxygen abundance in the ISM with time for the static cloudy model and the infall cloudy model is shown in Fig. 3, in comparison with the evolution of the reference diffuse model. The interaction of the clouds with the expanding supershell has a non-steady behavior, resulting in moments in which the attained average metallicity of the galaxy is larger than in the diffuse model and moments in which it is lower. As we have explained in the Introduction, this depends essentially on the competition between the dilution effect (due to the evaporation of the metal-free clouds, which tend to reduce the metallicity) and the drag effect (the delayed production of galactic outflows, which tends to increase the metallicity).

At the end of the simulation, however, the metallicity of the cloudy models is lower than the one of the diffuse model (by $\sim 0.1 \mathrm{dex}$ for the static cloudy model; $\sim 0.4$ dex for the infall cloudy one). This shows that over the long term, the dilution effect prevails over the restraint effect of the metal-enriched gas and the clouds have on average the effect of reducing the metallicity. This is caused by two facts: on the one hand, (as explained in Sect. 3.1) the interaction clouds-supershell distorts the supershell and produces fingers and tongues through which the metals present in the cavity can leave the galaxy, therefore the ejection efficiency of metals is not significantly reduced (in some case it is even larger) compared to the diffuse model. The second effect of metal dilution due to cloud evaporation becomes discernible by the tendency that in the infall cloudy model the oxygen abundance is continuously reduced with time after a maximum.

Models reproducing NGC1569 show the same kind of behavior explained above, therefore we do not describe them in this contribution. A comprehensive description of these models (and of others in which the IMF and the nucleosynthetic yields are varied) can be found in Recchi et al. (2006).

\section{Conclusions}

Although models of DG winds show strikingly that the low effective yield causes the low metallicity in dIrrs (Garnett 2002), nevertheless, it is obvious that a vertically extended gas disk and/or an enveloping gas reservoir is able to hamper the outflow of hot metal-rich supernova gas. The presented results demonstrate that the effects of existing embedded gas clouds and their evaporation on the effective yield is not straightforward predictable but depends on the porosity of the gas envelop and its reservoir, i.e. on the delivery of clouds, and must be studied in detail.

Due to dynamical processes and thermal evaporation, the clouds survive only a few tens of Myr. Due to the additional cooling agent, the energy of cloudy models is reduced by $20-40 \%$ compared with diffuse ones. The clouds delay the development of large-scale outflows, helping in retaining a larger amount of gas inside the galaxy. However,

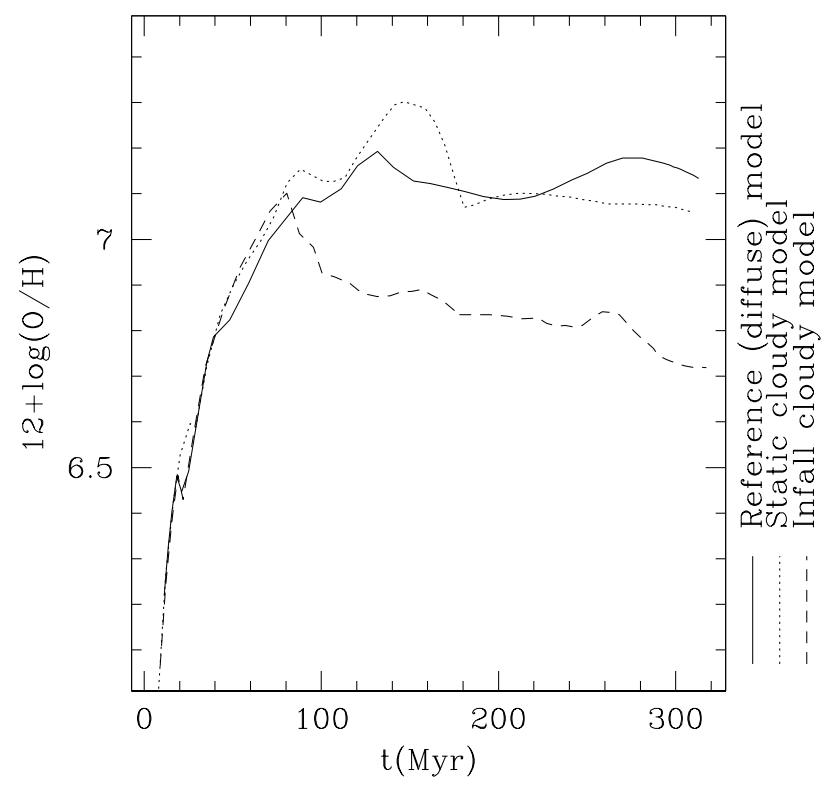

Fig. 3 Evolution of $12+\log (\mathrm{O} / \mathrm{H})$ for the static cloudy model (dotted line), the infall cloudy model (dashed line) and the reference diffuse model (solid line).

their bullet effect pierces the expanding supershell and creates holes through which the superbubble can vent out the freshly produced metals. The resulting final metallicity is generally smaller (by $\sim 0.4$ dex at most, depending on the model) than the one attained by diffuse models.

Nevertheless, models with self-regulated SF and selfconsistent treatment of the ISM, in particular with respect to cloudy and hot phases, are requested to evaluate the chemical abundances reliably.

Acknowledgements. The organizers of the Splinter Meeting are acknowledged for putting together a very enjoyable and interesting meeting. S.R. acknowledges financial support from the Deutsche Forschungsgemeinschaft (DFG) under grant HE 1487/28-1.

\section{References}

Cowie, L.L., McKee, C.F.: 1977, ApJ 211, 135

de Heij, V., Braun, R., Burton, W.B.: 2002, A\&A 391, 67

D’Ercole, A., Brighenti, F.: 1999, MNRAS 309, 941

Garnett, D.R.: 2002, AJ 581, 1019

Hensler, G.: 2003, ASP Conf. 304, eds. C. Charbonnel et al., p. 371

Hensler, G., Theis, Ch., Gallagher, J.S. III: 2004, A\&A 426, 25

Köppen, J., Hensler, G.: 2005, A\&A 434, 531

Recchi, S., Hensler, G.: 2007, A\&A 476, 841

Recchi, S., Hensler, G., Angeretti, L., Matteucci, F.: 2006, A\&A 445,875

Recchi, S., Matteucci, F., D’Ercole, A.: 2001, MNRAS 322, 800

Recchi, S., Matteucci, F., D’Ercole, A., Tosi, M.: 2004, A\&A 426, 37 (Paper I)

Samland, M., Hensler, G., Theis, Ch.: 1997, ApJ 476, 544 